\title{
Combining BCI with Virtual Reality: Towards New Applications and Improved BCI
}

Fabien Lotte, Josef Faller, Christoph Guger, Yann Renard, Gert Pfurtscheller, Anatole Lécuyer, Robert Leeb

\section{Introduction}

Historically, the main goal of Brain-Computer Interface (BCI) research was, and still is, to design communication, control and motor substitution applications for patients with severe disabilities [75]. These last years have indeed seen tremendous advances in these areas with a number of groups having achieved BCI control of prosthetics, wheelchairs and spellers, among other [49]. More recently, new applications of BCI have emerged that can be of benefit to both patients and healthy users alike, notably in the areas of multimedia and entertainment [51]. In this context,

Fabien Lotte

INRIA Bordeaux Sud-Ouest, 351 cours de la libération, F-33405, Talence, France. e-mail: fabien. lottedinria.fr

Josef Faller

Insitute for Knowledge Discovery, Laboratory of Brain-Computer Interfaces, Graz University of Technology, Krenngasse 37, A-8010 Graz, Austria. e-mail: josef . falleretugraz . at

Christoph Guger

g.tec medical engineering, Sierningstrasse 14, A-4521 Schiedlberg, Austria. e-mail: gugerligtec.at

Yann Renard

Independant Brain-Computer Interfaces Consultant, France e-mail: yann.renarde aliceadsl.fr

Gert Pfurtscheller

Insitute for Knowledge Discovery, Laboratory of Brain-Computer Interfaces, Graz University of Technology, Krenngasse 37, A-8010 Graz, Austria. e-mail: pfurtschel leretugraz . at

Anatole Lécuyer

INRIA Rennes Bretagne-Atlantique, Campus Universitaire de Beaulieu, F-35042 Rennes Cedex, France. e-mail: anatole. lecuyereinria.fr

Robert Leeb

Chair in Non-Invasive Brain-Machine Interface, École Polytechnique Fédérale de Lausanne, Station 11, CH-1015 Lausanne, Switzerland. e-mail: robert. leeb@epfl . ch 
combining BCI with Virtual Reality (VR) technologies has rapidly been envisioned as very promising $[37,40]$. Such a combination is generally achieved by designing a system that provides the user with immersive $3 \mathrm{D}$ graphics and feedback with which it can interact in real-time by using the BCI. The promising potential of this $\mathrm{BCI}-\mathrm{VR}$ combination is visible at two levels. On one hand, $\mathrm{BCI}$ is seen by the VR community as a new input device that may completely change the way to interact with Virtual Environments (VE) [37]. Moreover, BCI might also be more intuitive to use than traditional devices. In this sense, BCI can be seen as following a path similar to that of haptic devices a few years ago [7], that led to new ways of conceiving VR interaction. On the other hand, VR technologies also appear as useful tools for BCI research. VE can indeed be a richer and more motivating feedback for $\mathrm{BCI}$ users than traditional feedbacks that are usually in the form of a simple 2D bar displayed on screen. Therefore a VR feedback could enhance the learnability of the system, i.e., reduce the amount of time needed to learn the BCI skill as well as increase the mental state classification performance $[40,64]$. VE can also be used as a safe, cost-effective and flexible training and testing ground for prototypes of BCI applications. For instance, it could be used to train a patient to control a wheelchair with a BCI [39] and to test various designs for the wheelchair control, all of this without any physical risk and with a very limited cost. As such, VR can be used as an intermediary step before using BCI applications in real-life. Finally, VR could be the basis of new applications of BCI, such as 3D video games and artistic creation for both patients and healthy users, as well as virtual visits (cities, museums ...) and virtual online communities for patients, in order to address their social needs ${ }^{1}$.

Designing a system combining BCI and VR comes with several important challenges. First, the $\mathrm{BCI}$ being used as an input device, it should be, ideally, as convenient and intuitive to use as other VR input devices. This means that (1) the BCI should provide the user with several commands for the application; (2) the user should be able to send these commands at anytime, at will, i.e., the BCI should be self-paced (a.k.a. asynchronous); (3) the mapping between the mental states used and the commands (i.e., the interaction technique) should be intuitive, efficient, and not lead to too much fatigue for the user. This last point is particularly challenging since current BCI are usually based on a very small number of mental states, typically only 2 or 3 , whereas the number of interaction tasks that can be performed on a typical VE is very large, usually much larger than 3. From the point of view of the VE design and rendering, the challenges include (1) to provide a meaningful VR feedback to the user, in order to enable him to control the BCI; (2) to integrate the stimuli needed for BCI based on evoked potentials as tightly and seamlessly as possible in order not to deteriorate the credibility and thus the immersiveness of the VE and (3) to design a VR application that is useful and usable despite the huge differences between a typical VE and the standard $\mathrm{BCI}$ training protocols.

This chapter presents an overview of the research works that have combined BCI and VR and addressed these challenges. As such, (1) it surveys recent works that use $\mathrm{BCI}$ to interact with VE, (2) it highlights the critical aspects and solutions for the

\footnotetext{
1 See, for instance, the work achieved as part of the BrainAble project: http://www .
} brainable.org/ 
design of BCI-based VR applications and (3) it discusses the related perspectives. It is organized as follows: Section 2 provides some introductory material on VR and the way to interact with VE using a BCI. Then, Section 3 reviews existing BCIbased VR applications according to the different neurophysiological signals used to drive the BCI. More particularly, Section 3.1 discusses VR applications controlled with a motor imagery (MI)-based BCI, Section 3.2 those based on Steady State Visual Evoked Potentials (SSVEP) and Section 3.3 those exploiting a P300-based BCI. Then, Section 4 elaborates on the impact of VR on BCI use, notably in terms of BCI performance and user experience. Finally, Section 5 concludes the chapter.

\section{Basic principles behind VR and BCI control}

This section gives some insights about how VE can be controlled with a BCI. In the first subsection, VR is defined and the typical interaction tasks are described. The suitability of the different BCI neurophysioligical signals (MI, P300, SSVEP) for each interaction task is also briefly mentioned. In the second subsection, a general architecture for BCI-based VR applications is proposed. This architecture is illustrated with examples of existing VR applications using BCI as input device.

\subsection{Definition of Virtual Reality}

A VR environment can be defined as an immersive system that provides the user with a sense of presence (the feeling of "being there" in the virtual world [8]) by means of plausible interactions with a real-time simulated synthetic world [36]. Such plausible interaction is made possible thanks to two categories of devices: input and output devices. First, the user must be able to interact with the virtual world in real time. This is achieved by using input devices such as game pads, data gloves, motion tracking systems or, as described in this chapter, BCI. Second, the user must be provided with real time feedback about the virtual world state. To this end, various output devices are generally used to render the virtual world content, such as visual displays, spatial sound systems or haptic devices.

According to Bowman et al [6] typical interaction tasks with a 3D-VE can be described as belonging to one of the following categories:

- Object selection: it consists in selecting an object among those available in the virtual world, typically in order to subsequently manipulate it.

- Object manipulation: it consists in changing attributes of an object in the virtual world, typically its position and orientation or other properties such as appearance and size.

- Navigation: it consists in modifying the user's own position and orientation in the virtual world in order to explore it. In other words, navigation can be defined as moving around the VE and changing the current point of view. 
- Application control: it consists in issuing commands to the application, to change the system mode or to activate various functionalities, for instance.

All these categories of interaction tasks can be performed with a BCI. However, each BCI paradigm is more or less suitable for each category of interaction task. For instance, MI and SSVEP-based BCI are more suitable for navigation tasks and possibly object manipulation because they can issue commands continuously and potentially in a self-paced way. On the other hand, P300-based BCI let the user pick one item among a list of usually at least four, such command being issued in a discrete and synchronous way. For this reason, they are more suitable for object selection tasks. The suitability of each BCI paradigm is discussed more in details and illustrated in Sections 3.1, 3.2 and 3.3 respectively.

\subsection{General architecture of BCI-based VR applications}

Implementing a BCI control for a VR system can be seen as using the BCI as an input device to interact with the VE. Therefore, it consists in providing the user with a way to act on the virtual world only by means of brain activity, and using the available output devices to provide a meaningful feedback to the user. So far, only visual feedback has been deeply investigated in the context of BCI-based VR applications, but other modalities, in particular audio and haptics, would also be worth studying in the future. A BCI-based VR setup typically involves two independent softwares: 1) a BCI software to record brain signals, process them to extract relevant features and classify mental states in real-time in order to generate commands, and 2) a VR software to simulate and render a virtual world, provide feedback to user and process the received commands. Therefore, these two softwares must be able to communicate in order to exchange information and commands. Figure 1 provides a schematic representation of BCI control of a VR application.

In addition to these software considerations, there are also several hardware related issues that must be considered when using a BCI system in a VE: (1) the biosignal amplifiers must be able to work in such a noisy environment, (2) the recordings should ideally be done without wires to avoid collisions and irritations within the environment, (3) the BCI system must be coupled with the VR system to exchange information fast enough for real-time experiments and (4) in the case of CAVE systems, users mostly want to move around and therefore active EEG electrodes should be used to avoid movement artifacts.

In order to illustrate this general architecture implementation and propose a complete setup, we can mention two softwares which are devoted to BCI and VR as an example: OpenViBE and Ogre3D. OpenViBE ${ }^{2}$ is a free software platform to design, test and use BCI [63]. OpenViBE has been successfully used for the three major families of BCI: Motor Imagery [46], P300 [10] and SSVEP [44]. Ogre3D 3

\footnotetext{
2 http://openvibe.inria.fr/
}

${ }^{3}$ http://www.ogre3d.org 


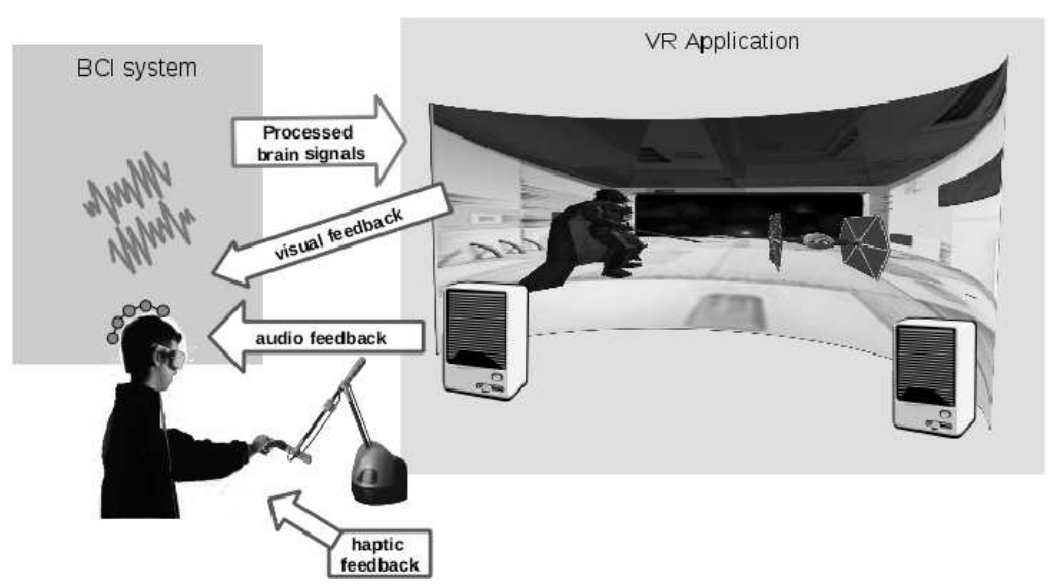

Fig. 1 General architecture of a BCI-based VR application: the user generates specific brain activity patterns that are processed by the BCI system and sent as command to the VR application. In return, the VR application provides meaningful feedback to the user, this feedback being potentially any combination of visual, audio or haptic feedback forms. This combination of "control on the VE" and "feedback from the VE" can elicit the sense of presence.

is a scene-oriented, flexible 3D engine that is capable of producing realistic representations of virtual worlds in real time. Ogre3D also includes extensions for spatial sound, physics simulation, etc. Moreover, it has been successfully used to simulate VE on equipments ranging from basic laptops to fully immersive systems such as CAVE systems [11]. These two softwares can communicate and exchange information, commands and responses using the Virtual Reality Peripheral Network (VRPN), a widely used library proposing an abstraction of VR devices [69]. Since both OpenViBE and Ogre3D have VRPN support, either natively or through contributions, they are able to communicate efficiently in order to design BCI-based VR applications. Those softwares have been used to design BCI-based VR applications such as those described in $[46,44,47]$. Generally not only VRPN, but any other interface (like proprietary TCP, UDP connections) can be used to communicate with existing VR systems.

Naturally, various other software and hardware can also be used to design BCIbased VR applications, such as Matlab/Simulink for real-time EEG signal processing and XVR (eXtremeVR 3D software, VRMedia, Italy) for VE design and applications $[28,27]$. Furthermore, simple projection walls with $\mathrm{Qt}^{4}$ application framework (Nokia Corporation, Finland), or stereoscopic presentation techniques such as head-mounted display (HMD) with VRjuggler, or even in fully-immersive multi-projection stereo-based and head tracked VE systems (commonly known as a "CAVE" [11] using DIVE software, or "DAVE" [21]) with the scene graph library OpenSG were already used and combined with a MATLAB based BCI [38]. On the

\footnotetext{
${ }^{4}$ http://qt.nokia.com/
} 
EEG hardware part, we can mention the gMOBIlab $+{ }^{5}$ (g.tec, Austria) which is a mobile EEG recording device that has been sucessfully used in VE (e.g., see [24]).

\section{Review of BCI-controlled VR applications}

This section reviews works that have used BCI to interact with VR applications. These works are arranged according to the neurophysiological signal used to drive the BCI: Motor Imagery (Section 3.1), SSVEP (Section 3.2) and P300 (Section 3.3). It should be mentioned that Section 3.1 describes more works than the other two sections, since more groups have used MI as the input signal to BCI-based VR applications. This is probably due to the fact that MI is a popular and well-studied neurophysiological signal for BCI [60], and that, contrary to SSVEP and P300, MI does not require any external stimulus which could be more convenient and natural for the the user of a VR application.

\subsection{Motor imagery controlled VR environments}

In this section we will focus on BCI based on MI, meaning on the analysis and classification of sensorimotor electroencephalographic (EEG) patterns generated during the imagination of specific movements (MI of left and right hand) [60, 59]. The imagination of different types of movements results in a characteristic change of the EEG over the sensorimotor cortex which is called event-related de/synchronisation [58]. After the computer learned the user-specific patterns, they can be used to control the movement of a bar to the right or left, just by imagining right or left hand movements. The same principle can be used to control simple movements in VEs.

The progress and comparison of MI-BCI controlled VR was first shown by Leeb and Pfurtscheller, by increasing the complexity of their studies from controlling a simple bar feedback in a synchronous manner till a self-paced (asynchronous) BCI in highly immersive VE [38]. In their first work, users perceived the feeling of rotating with constant speed to the right and left depending on the imagined hand movement (see Figure 2.a), while the rotation information was integrated over one trial [42]. Interestingly, no differences between HMD and CAVE feedback could be found, but all users performed better compared to standard bar feedback. The reason for the same VE performance was that users lost the spatial orientation while rotating, which disturbed them. In a similar experiment the imagination of foot movement was used to walk forward in a virtual street $[40,56]$. Correct classification of foot motor imagery was accompanied by forward movement at constant speed, whereas a correct classification of hand motor imagery stopped the motion.

\footnotetext{
5 http://www.gtec.at
} 
Incorrect classification of hand motor imagery resulted in backward motion (same speed) and incorrect foot in halting. The walking distance was scored as a "cumulative achieved mileage" (CAM, [40]), which was the integrated forward/backward distance covered during foot movement imagination and was used as performance measurement. All users achieved their best results within the CAVE and the worst in the standard BCI condition, so we can assume that the use of VR as feedback stimulated the participant's performances. The results indicate that foot motor imagery is a suitable mental strategy to control events within the VEs, because the imagination of feet movement is a mental task which comes very close to that of natural walking. It was observed that in the CAVE condition (highest immersion) the performance variation were stronger than in the control condition. One possible interpretation is that VR feedback amplifies both positive and negative feedback effects on the performance. The wrong behaving rich visual feedback can modify the EEG activity and thereby results in a further deterioration of performance [40].

The next important step was to overcome the cue-based interactions and to incorporate free will decisions (intentional control). Thereby users could navigate freely through a virtual apartment (see Figure 2.b), whereby at every junction the users could decide by their own, how they wanted to explore the VE [41]. The apartment (maze like) was designed similar to a real world application, with a goal-oriented task (predefined target room), a high mental workload and a variable decision period for the user. For comparison reasons, synchronous BCI sessions with a standard BCI bar feedback have been performed before and after the sessions with the virtual apartment, whereby the experiments with the virtual apartment were performed both in front of a normal TFT monitor and in an immersive VE. The users noted that the task in the apartment was much harder compared to the prior feedback training, because it was necessary not only to perform the "correct" imagination, but also the shortest way through the apartment had to be found. Therefore the cognitive load was much higher compared to the standard BCI paradigm. According to the hypothesis, it was found that the performance improves (decrease of error) over the sessions and the statistically significant lowest error could be found during the sessions with virtual feedback [41].

Giving the user the full control over timing and speed, was demonstrated in a study where users had to explore the Austrian National Library (see Figure 2.c). The participants were navigating down the library hall at their own pace but had to stop at several specific points (e.g. statue, column) [43]. After a variable pause time (between 20-95 seconds) the experimenter gave the command to restart moving. Navigating always happened when the users performed foot motor imagery. Seven users accomplished the study with a very small number of false positive. Most interestingly in this study are the extremely long periods (up to 1.5min) of pause times, where the user intentionally delivered no commands.

In their final study a tetraplegic patient used the imagination of his paralyzed feet to control forward movement of his wheelchair in VR [39]. The task was to go/move down a virtual street and stop at every avatar which was lined up along the street (see Figure 2.d). The patient achieved in some runs $100 \%$ performance and in average $90 \%$. This work demonstrated for the first time that a tetraplegic 
user, sitting in a wheel chair, could control his movements in a VE by the usage of a self-paced BCI based on one single EEG recording. It has to be mentioned that VEs are especially attractive for a person who is wheelchair-bound. First, simply using a VE can give such persons access to experiences that may be long forgotten (or which they have never had). The fact that the user could still perform feet motor imagery, years after an injury that rendered him unable to use his feet, is a testament to the plasticity of the human brain (similar to [34]).

Another BCI controlled wheelchair study was performed by Grychtol et al [25] with healthy users. Their results confirmed how voluntary behavioral modification brought about by VR feedback can help to improve the performance of a BCI system. VR feedback played an important role in the users' ability to learn and perform the activity well.
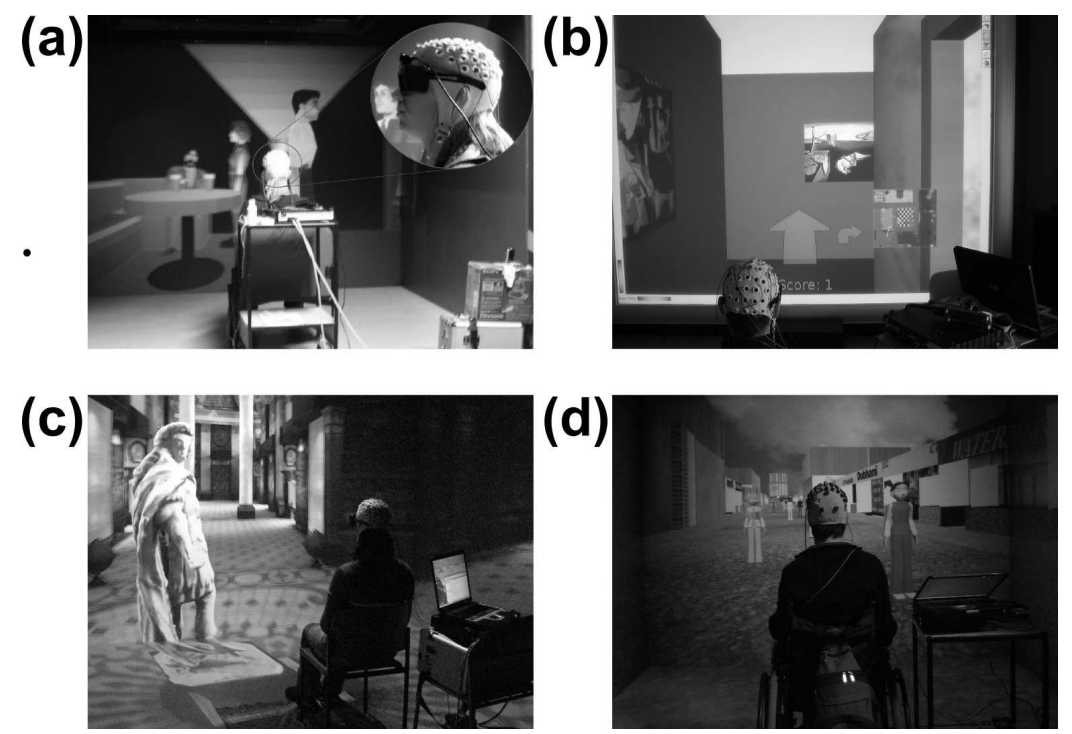

Fig. 2 Pictures of different MI-controlled VE: (a) exploring a virtual pub or (b) an apartment, (c) visiting the national library and (d) walking by thoughts in case of a wheelchair person.

While these applications are already very impressive and innovative, one could argue that most of them provide the user with only a single or two commands. This could be inconvenient for the user and restrict the range of possible VE the user could interact with. This has motivated some researchers to explore BCI-based VR applications providing a larger number of commands to the user. For instance, Scherer et al [66] proposed a 3-class self-paced BCI to freely navigate in a VE. With this BCI, the user could turn left, turn right or move forward by imagining a left hand, right hand or foot movement, respectively. While this proves to work and to be convenient for the user, this also highlighted some limitations of BCI-based interaction with VR. First, it stressed the well known performance problem of BCI, 
the performance being generally modest and decreasing when the number of classes to be identified increases [32]. Second, it suggested that performing navigation tasks in VR with a BCI can be tiring, especially when the user has to perform mental tasks continuously to go for one point to another or to keep the imagination over very long periods [43].

Some groups have recently proposed solutions to alleviate these issues, based on the use of specific interaction techniques. To address the limited classification performances of BCI system, the DIANA ${ }^{6}$ group proposed to navigate VE using a self-paced BCI based on 1 or 2 motor imagery tasks only [65, 72]. Indeed, with a number of classes as small as possible, the classification performances of the BCI are much more likely to be high. In order to still provide the user with 3 or more commands (in order to go forward, turn left or turn right) despite the BCI recognizing only 1 or 2 motor imagery states, they proposed specific interaction techniques. These techniques are based on a scanning principle (similar to the hex-o-spell [74]). This means that to select a given interaction command, the user had to perform a motor imagery task during a given time frame, each frame being associated to a different command. Their evaluations showed that, with this approach, users can actually freely navigate in a VE with a simple brain-switch [72].

In order to alleviate the fatigue caused by BCI-based navigation in VR, as well as to efficiently use the small number of MI tasks recognized by a BCI, INRIA ${ }^{7}$ also proposed a new interaction technique for BCI-based VR applications [46]. This technique, based on a 3-class self-paced BCI, provides the user with high-level commands, thus leaving the application in charge of performing the complex and tedious details (low-level aspects) of the interaction task. Thus, this can be seen as a form of shared-control [52]. The user can explore the VE by selecting points of interest such as navigation points (e.g., junctions, room entrances, etc.) or artworks. Interestingly enough, these navigation points can be generated completely automatically from the geometry of the VE. The user can select these points due to a sequence of binary choices. In addition to the two commands used to perform these binary choices, the user can use a third command to cancel any of his/her choice. Once a navigation point has been selected, the application takes all the necessary actions to perform the interaction task such as moving from the current navigation point to the next selected one. Evaluations, performed in the context of the exploration of a virtual museum (see Figure 3), showed that with this approach, users can navigate from one room to the other nearly twice as fast as with low-level commands, and with less fatigue.

Due to the huge potential of BCI-based VR applications, not only for patients but also for healthy users, it quickly became necessary to evaluate them outside laboratories, in close to real-life conditions. Such an evaluation was performed with the "use-the-force" application [47], a BCI-based VR game inspired by the Star Wars $^{\mathrm{TM}}$ movie. With this game, users were asked to control the takeoff of a virtual spaceship by using real or imagined foot movements (see Figure 4). The system

${ }^{6} \mathrm{http}: / / \mathrm{www}$.diana.uma.es

${ }^{7}$ http://www.inria.fr/en/ 


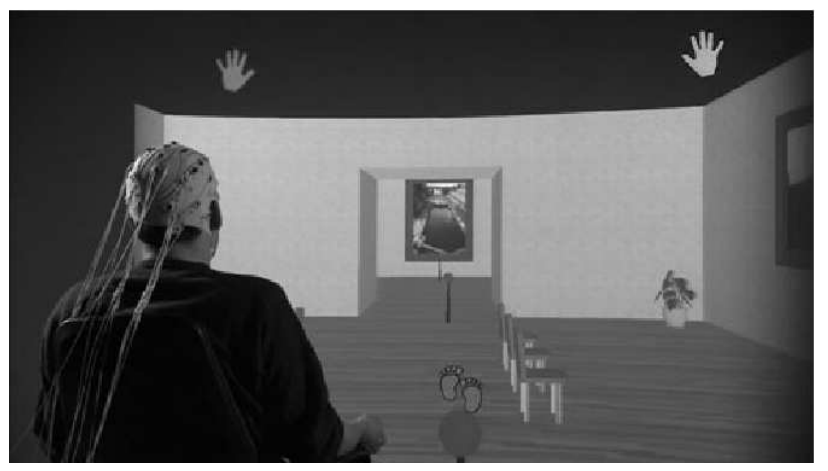

Fig. 3 Exploring a virtual museum using a BCI and high-level commands [46].

relied on a simple brain switch that detects the beta rebound posterior to the real or imagined foot movement, at electrode $\mathrm{Cz}$. The game was evaluated with 21 naïve users, during a public exhibition dedicated to VR. Despite the simplicity of the BCI design and the noisy environment, results showed that, without training, half the users could control the virtual object's motion by using real foot movements. A quarter of them could do so by using imagined foot movements. Furthermore, the whole application appeared enjoyable and motivating to the users.

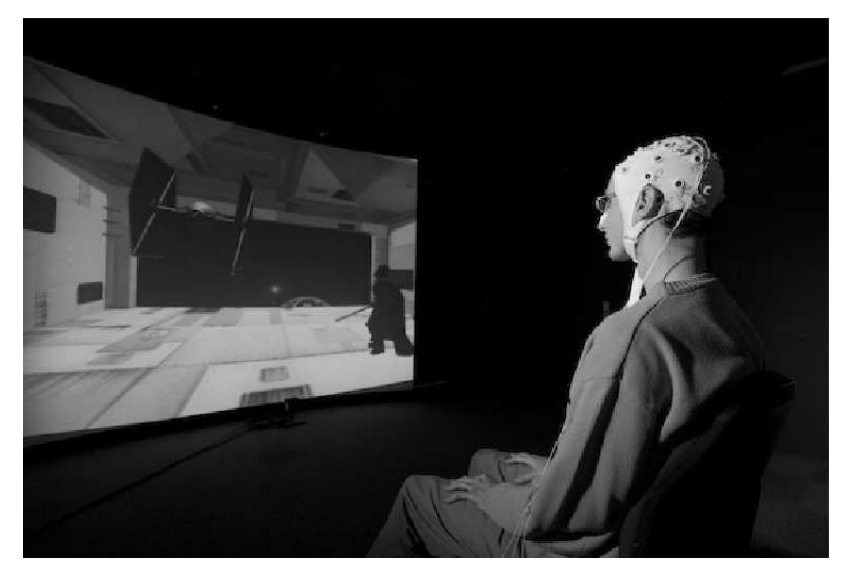

Fig. 4 The "Use-the-force" entertaining application [47], which enables its users to lift a virtual spaceship by using the BCI (C) Hubert Raguet/Phototheque CNRS).

Another instance of BCI to interact with complex applications was shown by Scherer et al [67]. The Brainloop interface provides a new way to interact with complex programs like Google Earth ${ }^{\mathrm{TM}}$. Thereby through remapping of commands and options the interface can be customized. In this study, a multi-level selection 
process and the use of mental tasks in parallel enabled the user to send multiple commands to the application.

Although not based on motor imagery, another BCI-based VR application deserves to be mentioned here since it was also evaluated in public places, outside the lab: the "AlphaWoW" application [51]. In this game, based on World Of Warcraft ${ }^{\circledR}$, the player controlled his avatar using a classical keyboard but can turn it from a fragile elf to a powerful bear by using a BCI. More particularly, the avatar shape (bear or elf) depended on the band power in the alpha band $(8-12 \mathrm{~Hz})$, the alpha rhythm power being related to the player's state of relaxation. In other words, when the player was stressed the avatar changed into a bear, and he/she has to relax to turn back the avatar into an elf. The evaluations showed that the game was received very positively despite the modest BCI performances, which were considered by the players more as a challenge than as a shortcoming. These different close-toreal-life-studies thus highlight the potential of BCI-based VR application and the need to push research efforts in these directions [51, 45, 67]

Table 1 Summary of BCI-based VR applications using Motor Imagery

\begin{tabular}{|c|c|c|c|c|c|}
\hline $\begin{array}{c}\text { interaction } \\
\text { task }\end{array}$ & $\begin{array}{c}\text { number of } \\
\text { MI tasks }\end{array}$ & $\begin{array}{c}\text { number of } \\
\text { commands }\end{array}$ & $\begin{array}{c}\text { synchronous } \\
\text { or self-paced }\end{array}$ & VE & Reference \\
\hline \hline navigation & 2 & 2 & synchronous & $\begin{array}{c}\text { exploring a } \\
\text { virtual pub }\end{array}$ & {$[38]$} \\
\hline navigation & 2 & 2 & synchronous & $\begin{array}{c}\text { navigating along a } \\
\text { virtual street }\end{array}$ & {$[40,56]$} \\
\hline navigation & 2 & 2 & synchronous & navigating & {$[25]$} \\
\hline navigation & 2 & 2 & $\begin{array}{c}\text { semi } \\
\text { synchronous }\end{array}$ & $\begin{array}{c}\text { exploring a } \\
\text { virtual apartment }\end{array}$ & {$[41]$} \\
\hline navigation & 1 & 1 & self-paced & $\begin{array}{c}\text { exploring a } \\
\text { virtual library }\end{array}$ & {$[43]$} \\
\hline navigation & 1 & 1 & self-paced & $\begin{array}{c}\text { moving along a } \\
\text { virtual street }\end{array}$ & {$[39]$} \\
\hline navigation & 3 & 3 & self-paced & $\begin{array}{c}\text { exploring the } \\
\text { "free-space" }\end{array}$ & {$[66]$} \\
\hline navigation & $1-2$ & 4 & self-paced & $\begin{array}{c}\text { exploring a maze } \\
\text { or park }\end{array}$ & {$[65,72]$} \\
\hline $\begin{array}{l}\text { navigation } \\
+ \text { selection }\end{array}$ & 3 & more than 3 & self-paced & $\begin{array}{c}\text { exploring a } \\
\text { virtual museum }\end{array}$ & {$[46]$} \\
\hline manipulation & 1 & 1 & self-paced & $\begin{array}{c}\text { lifting a } \\
\text { virtual spaceship }\end{array}$ & {$[47]$} \\
\hline $\begin{array}{l}\text { navigation } \\
+ \text { selection }\end{array}$ & 3 & more than 3 & self-paced & $\begin{array}{c}\text { controlling } \\
\text { Google Earth }\end{array}$ & {$[67]$} \\
\hline
\end{tabular}

Table 3.1 summarizes the numerous studies presented in this section, by describing some key characteristics of these BCI-based VR applications using MI. Several interesting points come out of this table. First, this table highlights the importance of self-paced BCI design for VR application in general and for navigation tasks in particular. Indeed, navigation is inherently a self-paced task. Moreover, despite the fact 
that self-paced BCIs are a challenging research topic that is not well explored [48], most of the aforementioned studies have designed and used such a BCI. Another point to notice is that, although most BCI-based VR applications provide the user with as many commands as MI tasks used, it is possible to provide more commands than MI tasks by using appropriate interaction techniques. Finally, this table stressed that MI has been almost exclusively used to perform navigation tasks in VR. Indeed, MI appears as particularly suitable for such a task since it enables spontaneous and self-paced control, which navigation should be. On the contrary, it is not convenient to perform selection tasks with MI, since MI provides only a few mental states and thus a few commands whereas virtual objects to be selected can be potentially numerous. As such, and as it will be highlighted in subsequent sections, BCI based on Evoked Potentials (SSVEP, P300) are more suitable for selection tasks since they can use numerous stimuli and corresponding brain responses.

\subsection{SSVEP based VR/AR environments}

A SSVEP is an electroencephalographic response occurring when a user perceives a visual stimulus flickering at a constant frequency [73]. This response is observed over the visual cortex (occipital electrodes), and consists of an EEG pattern oscillating at the same frequencies that the flickering stimulus and its harmonics. Interestingly enough, SSVEP can be modulated by attention, which means that the SSVEP response to a given stimulus will be stronger (i.e., with a larger amplitude) when the user focuses his/her attention on this stimulus.

Lalor et al [35] were the first to use an SSVEP-based BCI to control a character in a $3 \mathrm{D}$ gaming environment. In this game, a monster went from platforms to platforms by moving along a tight rope. From time to time, the monster lost its balance, and the user had to restore it by using the BCI. To do so, two flickering checkerboard were placed on each side of the VE, in order to elicit SSVEP at different frequencies. When the system detected that the user was focusing on the left or right checkerboard, it restored the monster's balance towards the left or right respectively. Later, Touyama worked towards more immersive applications based on SSVEP and showed that they could be used to change the point of view towards the left or right in a VE displayed in a CAVE-like system [70].

The works mentioned above proved that SSVEP-based BCI is a suitable and efficient way to interact with VE. One of its limitations though, is that it requires flickering stimuli in order to be used. In the context of VR applications, this has been mainly achieved by relying on flickering squares or checkerboards statically overlayed over the screen. As a consequence, the VE may look unnatural and is unlikely to elicit a strong sense of presence for the user. In order to address these limitations, Faller et al [20] presented a desktop-based virtual environment, where the stimuli were tightly integrated within 3D scenarios that allowed controlling avatar interaction and navigation. In one of the scenarios, seven healthy volunteers successfully controlled an avatar to alternately push one of two buttons in an asynchronous 
paradigm. The stimuli were fixed to the hands and hence dynamically following every avatar movement.

In another of the presented scenarios [20], five out of seven users successfully navigated an avatar in third person perspective through the same apartment scenario (see Figure 5.a) as presented in Leeb et al [41]. They could guide the avatar in discrete steps and turns by visually fixating one of three SSVEP stimuli that were fixed to the avatars back. Each successful classification would then trigger one of three associated navigation commands, go one step ahead, turn left $45^{\circ}$ or turn right $45^{\circ}$ (see Figure 5.b).

(a)

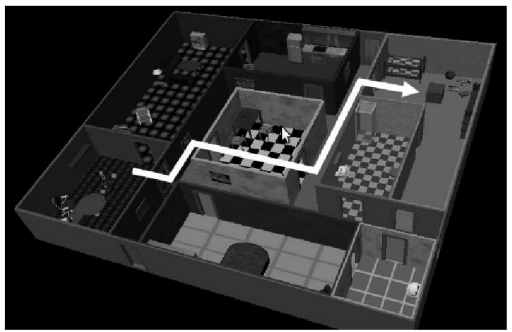

(b)

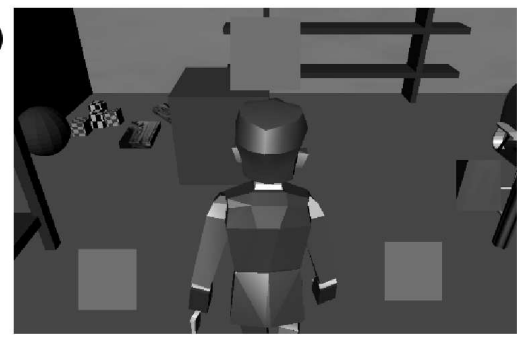

Fig. 5 Panel (a) shows an overview of the apartment scenario. The users were instructed to navigate the avatar along the path that is depicted by the white arrow. Panel (b) is a screenshot from an actual online scene, where the user navigates the avatar in third person perspective [20].

Still dealing with the integration of SSVEP-stimulus within VE, Legeny et al worked towards an even more natural and ecological approach [44]. In their work, which aimed at navigating in a virtual forest, the flickering stimuli necessary for SSVEP generation were displayed on butterfly wings. Three of these butterflies were displayed on screen, flying up and down in front of the user (see Figure 6). The user had to focus his/her attention on the left, center or right butterfly in order to go left, forward or right, respectively. The butterflies' antennas were also used to provide feedback to the user. Indeed, the further apart the two antennas of a butterfly were, the more likely this butterfly will be selected by the classifier as the one the user pays attention to. Such stimuli are therefore more naturally incorporated into the VE, and formal evaluations suggested that it indeed increased the subjective preferences and feeling of presence of the users.

Finally, moving beyond traditional VE, Faller et al $[18,19]$ extended their previous work into a SSVEP BCI system that relies on stimuli that are presented within immersive virtual and more interestingly, in an Augmented Reality (AR) environments. In a pilot study, three healthy volunteers were able to successfully navigate an avatar through an immersive VR slalom scenario based on embedded SSVEP stimuli. The complete scene was presented using a head-mounted display (HMD). Two of these three volunteers also succeeded in the immersive AR condition, where a camera was mounted on the HMD and the slalom scenario 3D graphics were injected into the live, real-world video by tracking fiducial markers (see Figure 7). 


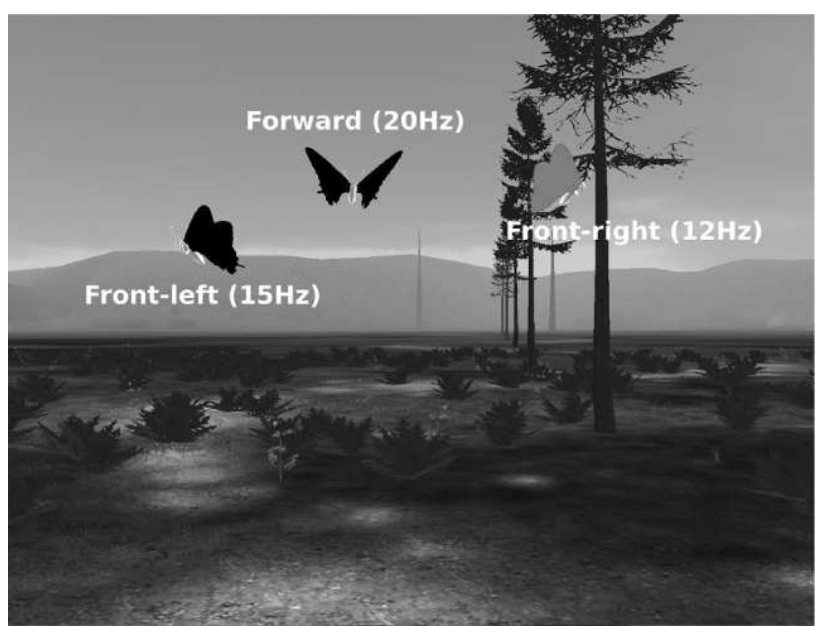

Fig. 6 Mimetic integration of SSVEP stimulation and feedback in a Virtual Environment [44]. The butterfly wings are flickering at different frequencies in order to enable SSVEP-based BCI control, while their antenna positions represent the real-time feedback, i.e., the butterfly the most likely selected by the user according to the classifier.
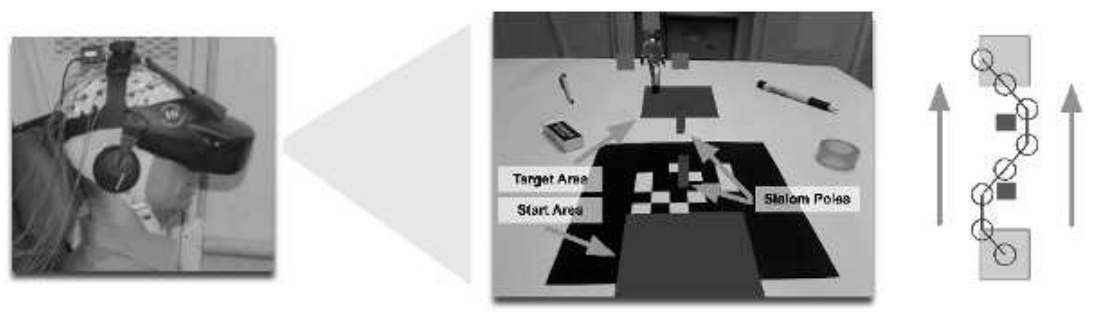

Fig. 7 The middle picture shows a screenshot of how the user saw the scene through the HMD seen in the left picture. The 3D graphics were tracked to the underlying fiducial marker. The participants were instructed to navigate the avatar through the slalom like in the picture on the right $[18,19]$.

The positive results from this first feasibility study suggest that AR SSVEP BCIs have the potential to vastly improve real-world practicality and usability of BCI systems by compensating for some of their traditional shortcomings such as the low bandwidth, by offering a richer, more direct, and intuitive interface. This would allow for a more goal-directed and seamless real-world interaction. In an AR SSVEP BCI system, stimuli targets can be spatially associated to distinct points of interest in the physical world. These may be abstract or may overlap physical objects such as devices, people or controls, which is an elegant and intuitive way of presenting the user with all possible interaction options. These systems could provide patients with a higher degree of self autonomy and functional independence by introducing more intuitive and effective smart home control. Apart from that, AR SSVEP BCIs can further introduce a valuable, additional communication or control channel for 
user groups that require or benefit from hands free operation like pilots, car-drivers or office workers.

\subsection{P300 based VR control}

One of the first efforts to combine VR and BCI technologies was achieved by Bayliss and Ballard [4, 3], and made use of the P300 evoked potential. The P300 is a positive waveform occurring roughly $300 \mathrm{~ms}$ after a rare and relevant stimulus $[75,17]$. In order to use a P300-based BCI, users have to focus their attention on a given stimulus randomly appearing among many others, each stimulus corresponding to a given command [14]. The appearance of the desired stimulus being rare and relevant, it is expected to trigger a P300 in the user's brain activity. In their study, Bayliss introduced a simple virtual smart home in which users could control different appliances (e.g., a TV or a light) using the P300-based BCI. 3D spheres were randomly appearing over the objects that can be manipulated and the user could turn them on or off simply by counting the number of times a sphere appears over the desired object.

More recently, a more interactive and richer virtual version of a smart home was implemented [24]. This smart home consists of a living room, a kitchen, a sleeping room, a bathroom, a floor and a patio as shown in the right side of Figure 8. Each room has several devices that can be controlled: TV, MP3 player, telephone, lights, doors, etc. Therefore, all the different commands were summarized in 7 control masks: a light mask, a music mask, a phone mask, a temperature mask, a TV mask, a move mask and a go to mask. The left side of figure 8 shows the TV mask and as an example the corresponding XVR image of the living room [15]. The user can e.g. switch on the TV by looking first at the TV symbol. Then, the station and the volume can be regulated. The bottom row of Figure 8 shows the go to mask with an underlying plan of the smart home. Inside the mask, there are letters indicating the different accessible spots in the smart home which flash during the experiment. Therefore, the user has to focus on the spot where he wants to go. After the decision of the BCI system, the VR program moves to a bird's eye view of the apartment and zooms to the spot that was selected by the user. This is a goal oriented BCI control approach, in contrast to MI navigation task, where each small navigational step is controlled. Experiments with 3 users yielded accuracies of the BCI system between 83 and $100 \%$ and showed that such a BCI system can be used for the smart home control [29]. For comparison a group study with healthy people with the standard P300 speller gave an average accuracy of $91 \%$ [26]. The Virtual Reality approach is a very cost effective way for testing the smart home environment together with the $\mathrm{BCI}$ system. Currently the BCI technology is interfaced to real smart home environments within the EC project SM4all ${ }^{8}$. The project aims at studying and developing

\footnotetext{
$\overline{{ }^{8} \text { http://www.sm4all-project.eu }}$
} 
an innovative middleware platform for inter-working of smart embedded services in immersive and person-centric environments [27].
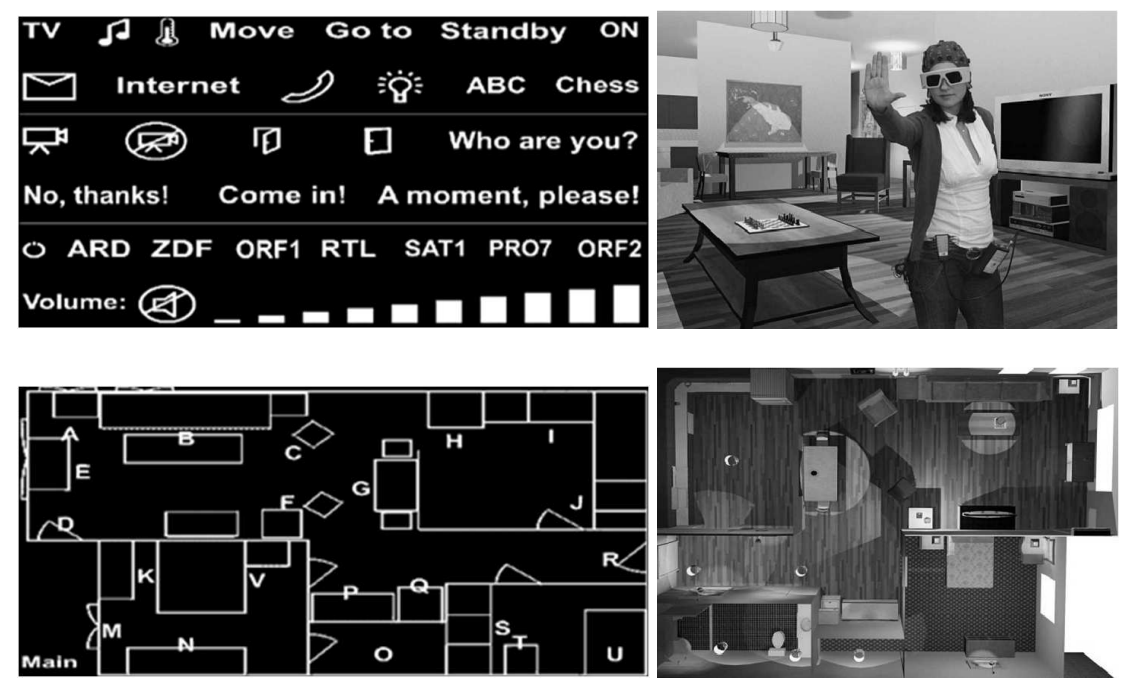

Fig. 8 Top left: Smart home control icons for TV, Telephone,... Top right: VR representation of the living room $[29,15]$. Bottom: Control icons to move to a certain position inside the apartment and corresponding bird eyes view of the apartment.

These different experiments yielded 2 important new facts for P300-based BCIs: (1) instead of displaying characters and numbers to the user, it appears that different icons can be used as well, (2) the BCI system does not have to be trained on each individual character. The BCI system was trained with EEG data of the spelling experiment and the user specific information was used also for the smart home control. This allows using icons for many different tasks without prior time consuming and boring training of the user on each individual icon. This reduces the training time in contrast to other BCI implementations were hours or even weeks of training are needed [5, 30, 71], which might be important for locked-in and ALS patients who have problems with the concentration over longer time periods. The P300 concept works also better if more items are presented in the control mask as the P300 response is more pronounced if the likelihood that the target character is highlighted drops down [33]. This results of course in a lower information transfer rate, but enables to control almost any device with such a BCI system. Especially applications which require reliable decisions are highly supported. Therefore the P300 based BCI system enables an optimal way for the smart home control. In a further study the P300 smart home control was combined with a head tracker to switch on and off the BCI system. This means if the person was looking at the BCI system then it was switched on and a selection could be done. If the person turned to the VR projected the BCI system was switched off. Recently a hybrid version of 
a P300 and SSVEP BCI was used for controlling the smart home environment. The P300 BCI was used to select the command and SSVEP was used to switch on and off the BCI system [16]. These hybrid BCI systems hence demonstrated that BCI could be used practically to interact with a virtual smart home, hence potentially offering new and promising applications for patients, at home. It is worth mentioning that Groenegress compared the P300-based BCI control with a gaze-based selection method coupled with wand navigation [24]. Results suggested that the P300 BCI gives lower Presence scores which might be due to the lack of motor actions which are relevant for semantic tasks and more breaks in presence.

\section{Impact of Virtual Reality on BCI}

In contrast to traditional interfaces like mouse or keypad, BCI systems could potentially promise a more direct and intuitive way of interacting and thereby overcome some limitations of navigating within VEs [68]. This is especially obvious for stimulus-dependent BCI-VR systems, where users can control appliances in the VE by simply directing their eye gaze and/or focus of attention towards the desired element (e.g., looking at the TV to switch it on, looking at the door to open it [1]). On the other hand motor imagery offers an intuitive way of VE control, for example, imagining foot movements for moving forward in a VE [43, 56]. This could overcome the problem of the contradictory stimuli while navigating VEs using a hand-held device and the reduced sense of being present in the VE [68]. On the other hand it is well known that feedback is one of the key components of a BCI, as it provides the user with information about the efficiency of his/her strategy and enables learning. The studies mentioned above show realistic and engaging VR feedback scenarios, which are closely related to the specific target application. However, the processing of such a realistic feedback stimulus may also interfere with the motor imagery task, and thus might impair the development of BCI control [50]. Furthermore, characteristic EEG changes during VE conditions were reported in [56], where a dominant ERS pattern which was permanently present in the CAVE, was less pronounced in the HMD and not existing at all in the normal feedback. Nevertheless, it was presented in Section 3.1 that VR improves the BCI performance; either the users achieved their best results within VR [40, 56, 25] compared to normal feedback or even $100 \%$ performance result in VR [39] or achieved the lowest error with virtual feedback [41]. Generally it can be stated that VR feedback amplifies both positive and negative feedback effects on the performance.

Besides BCI performances, other data can also be used to investigate the influence and impact of VR on the BCI. For most of the MI studies mentioned in the beginning of Section 3.1, the electrocardiogram was recorded in addition and questionnaires were conducted. An interesting aspect is that mental simulation of a movement (motor imagery) results in cardiovascular changes explained by two factors: anticipation of movement and central preparation of movement [12, 53]. The heart rate (HR) generally decreases during motor imagery in normal BCI conditions 
(without VR feedback) $[38,57]$ which is similar to that observed during preparation for a voluntary movement. In case of VR feedback, the HR can be increased during effortful imagery $[57,55,38]$. The heart rate acceleration in the VE is interpreted as effect of an increased mental effort [13] to move as far as possible in VE. This underlines the importance of VR feedback in modifying emotional experiences and enhances autonomic and visceral responses. The HR changes can be in the order of several beats-per-minute (bpm) and therefore could be used to increase the classification accuracy of an ERD-based BCI when both the EEG and the HR are analyzed simultaneously [54].

These heart rate changes were found in most studies of Section 3.1: (1) In the "walking from thought" study [56], instead of the normal decrease of 3-5\%, an increase of up to $5 \%$ was found. Furthermore, the results provide provisional evidence that moving backwards (negative feedback) resulted in a stronger and longerlasting HR increase than forward moving (positive one) [57]. (2) In the virtual apartment study [41] the analysis of the heart rate showed that during the BCI condition a preparatory HR deceleration could be found, which is in line with our prior study [57] but not in the VE conditions since no preparation cue was provided. Generally, the visible HR deceleration is stronger for good trials than for bad trials in all conditions (with removed preparatory phase). Furthermore, a better classification accuracy was accompanied with a stronger deceleration [38]. (3) In contrast to these results, HR increases are observed for two users during VE feedback in study [57]. Interestingly, the slight HR increase $(0.5-1 \%)$ before the cue in the VR feedback conditions could be the effect of the anxiety of the user to find the best and correct way for the next decision. Moreover this increase is more dominant in the immersive VE condition, which correlates with the reported higher motivation. (4) In the case of the self-paced navigating study inside the virtual library [43], a phase relationship between the HR and the EEG could be identified. Movement onset occured during periods of increasing HR, only one user showed a not statistically significant decreasing HR [38]. Although the users were participating in a self-paced experiment, the performance was not completely self-paced but aligned with the underlying cardio-vascular pace. (5) Finally, in the study with the tetraplegic patient [39], the analysis revealed that the induced beta oscillations were accompanied by a characteristic heart rate (HR) change in form of a preparatory HR acceleration followed by a short-lasting deceleration in the order of 10-20 bpm [55]. This provides evidence that mental practice of motor performance is accompanied not only by activation of cortical structures but also by central commands into the cardiovascular system with its nuclei in the brain stem. Another reason for the observed preparatory HR increase could be that the tetraplegic patient was highly motivated and therefore directed increased attention to "walk" in the immersive environment.

Summing up, the use of VR enhanced the user's BCI and application performances and provided motivation (see [40, 56, 38, 64, 61]). These findings are supported by the outcome of the questionnaires and heart rate analysis, where the users self-rated their success stronger than their failure and a stronger HR decrease could be found as well for good classification results. Especially the HR outcome, in the case of an asynchronous (self-paced) BCI, was interesting and it can be speculated 
that the "free will" of the users was affected by processes operating automatically and unconsciously [31]. Similar influences on self-paced hand movements without awareness of the participants can be caused by transcranial magnetic stimulation [2].

\section{Conclusion}

In this chapter, we have presented and discussed how BCI and VR could be combined and surveyed the related works. As a summary, recent works have shown that BCI could be used to navigate virtual worlds, mostly thanks to motor imagery and SSVEP-based BCI, since these signals enable continuous and self-paced control. BCI could be used to select and manipulate virtual objects as well, for which evoked potentials (P300, SSVEP) seem to be the most used and probably the most appropriate neurophysiological signals. Indeed, such signals enable to select objects simply by paying attention to the corresponding stimulus, and a BCI can deal with numerous such stimuli. On the contrary, MI-based BCI can use only a limited number of mental tasks and are thus less suitable for tasks involving the selection and/or manipulation of numerous virtual objects. These works have also highlighted the challenge in designing BCI-based VR applications, BCI control being usually slow, error-prone and with limited degrees of freedom whereas a VE can be highly interactive and complex. In this context, the design of appropriate interaction techniques and paradigms has shown to be a suitable way to alleviate these limitations and should thus be further studied. Finally, this chapter has highlighted that not only BCI can be a useful interaction device for VE, but that VR could also be a useful technology for BCI. In particular, VR being a rich and motivating environment for the BCI user, it has been shown that this could lead to improved BCI performances, higher motivation and engagement, and reduced human training time in comparison to classical feedback forms. Therefore, BCI and VR can certainly be seen as complementary tools, BCI being useful as an interaction device to enhance the VR experience, and VR being an environment that benefits BCI research and performances.

The various works described in this chapter have also opened the doors to exciting and promising new research topics to further develop the connection between BCI and VR. Indeed, it would be interesting to study how BCI could be used more naturally, transparently and ecologically with virtual environments, in order to make the interactive experience even more immersive. In addition to the classical need for BCI with higher recognition performances, it would be interesting to study whether new mental states and neurophysiological signals could be used to drive a BCI more naturally within a VE. For instance, a study by Plass-Oude Bos et al suggested that visual spatial attention could be detected, to some extent, from EEG signals and could thus be used in the future to naturally look around in a VE [62]. Such kind of research efforts should be encouraged in order to develop the repertoire of mental states that could be used to interact mentally with VE. Similarly, further research in the area of passive BCI $[23,76]$ could help to monitor different mental states of 
the user (e.g., flow, presence, emotions, attention, etc.) and dynamically adapt the content of the VE accordingly, thus providing an enhanced experience for the user. Since it has been shown that VR could lead to enhanced BCI performances, it would also be interesting to further study the impact of various VR feedback forms (e.g., visual, tactile or audio) on BCI, in order to identify how VR technologies can best optimize the performance and learnability of the system. The specificity of BCI, which do not rely on peripheral nerves and muscles contrary to tradional interfaces, also raise some interesting questions (and maybe answers) related to embodiement and embodied cognition. As such, a system combining BCI and VR might prove a worthy tool and research topic for philosophy and cognitive sciences [9]. Finally, using BCI to interact with VE has the potential to lead to several practical and useful applications. For patients, BCI-based VR applications could enable them to have access to entertainment (e.g., 3D video games), art and culture (e.g., digital creation of paintings, virtual visits of museums and cities) as well as a better social life (e.g., with virtual online communities), which their disabilities might prevent them from doing. This will enable BCI to be useful beyond restoring mobility and basic communication by addressing other important needs of patients [77]. For healthy users, BCI-based VR applications could also be useful, in areas such as entertainment as well [51] - although this may require more improvements in BCI design [45] and artistic expression [22]. In short, it appears that combining BCI and VR is a promising research topic that is worth being further explored.

Acknowledgements This work was supported by the European Union projects PRESENCIA (IST-2001-37927) and PRESENCCIA (IST-2006-27731), furthermore by the French National Research Agency projects OpenViBE (ANR-05-RNTL01601) and OpenViBE2 (ANR-09-CORD017).

\section{References}

1. Allison, B.Z., McFarland, D.J., Schalk, G., Zheng, S.D., Jackson, M.M., Wolpaw, J.R.: Towards an independent brain-computer interface using steady state visual evoked potentials. Clin Neurophysiol 119(2), 399-408 (2008)

2. Ammon, K., Gandevia, S.C.: Transcranial magnetic stimulation can influence the selection of motor programmes. J Neurol Neurosurg Psychiatry 53(8), 705-7 (1990)

3. Bayliss, J.D.: Use of the evoked potential P3 component for control in a virtual apartment. IEEE Trans Neural Syst Rehabil Eng 11(2), 113-116 (2003)

4. Bayliss, J.D., Ballard, D.H.: A virtual reality testbed for brain-computer interface research. IEEE Trans Rehabil Eng 8(2), 188-90 (2000)

5. Birbaumer, N., Ghanayim, N., Hinterberger, T., Iversen, I., Kotchoubey, B., Kübler, A., Perelmouter, J., Taub, E., Flor, H.: A spelling device for the paralysed. Nature 398, 297-298 (1999)

6. Bowman, D., Kruijff, E., Jr., J.L., Poupyrev, I.: 3D User Interfaces: Theory and Practice. Addison-Wesley/Pearson Education (2005)

7. Burdea, G.: Force and touch feedback for virtual reality. John Wiley \& Sons (1996)

8. Burdea, G., Coiffet, P.: Virtual Reality Technology. John Wiley and Sons (2003)

9. Clark, A.: Supersizing the mind: Embodiment, action, and cognitive extension. Oxford University Press, USA (2008) 
10. Congedo, M., Goyat, M., Tarrin, N., Varnet, L., Rivet, B., Ionescu, G., Jrad, N., Phlypo, R., Acquadro, M., Jutten, C.: "Brain Invaders": a prototype of an open-source P300-based video game working with the OpenViBE platform. In: 5th International BCI Conference (2011)

11. Cruz-Neira, C., Sandin, D., Defanti, T., Kentyon, R., Hart, J.: The CAVE : audio visual experience automatic virtual environment. Communications of the ACM 35(6), 64-72 (1992)

12. Damen, E.J., Brunia, C.H.: Changes in heart rate and slow brain potentials related to motor preparation and stimulus anticipation in a time estimation task. Psychophysiology 24(6), 700$13(1987)$

13. Decety, J., Jeannerod, M., Germain, M., Pastene, J.: Vegetative response during imagined movement is proportional to mental effort. Behavioural Brain Research 42, 1-5 (1991)

14. Donchin, E., Spencer, K.M., Wijesinghe, R.: The mental prosthesis: assessing the speed of a P300-based brain-computer interface. IEEE Transactions on Neural Systems and Rehabilitation Engineering 8, 174-179 (2000)

15. Edlinger, G., Holzner, C., C.Groenegress, Guger, C., M.Slater: Goal-oriented control with brain-computer interface. In: Lecture Notes in Computer Science 5638, pp. 732-740 (2009)

16. Edlinger, G., Holzner, C., Guger, C.: A hybrid brain-computer interface for smart home control. In: Human Computer Interface Conference (2011)

17. Elshout, J., Molina, G.G.: Review of brain-computer interfaces based on the P300 evoked potential. Tech. Rep. PR-TN 2009/00066, Koninklijke Philips Electronics (2009)

18. Faller, J., Allison, B., Brunner, C., Schmalstieg, D., Pfurtscheller, G.: A software SSVEP $\mathrm{BCI}$ integrating stimuli within motivating and immersive virtual and augmented reality environments. In: Real Actions in Virtual Environments (RAVE) conference, Barcelona, Spain. (2010)

19. Faller, J., Leeb, R., Pfurtscheller, G., Scherer, R.: Avatar navigation in virtual and augmented reality environments using an SSVEP BCI. In: International Conference on Applied Bionics and Biomechanics (ICABB) 2010, Venice, Italy (2010)

20. Faller, J., Müller-Putz, G.R., Schmalstieg, D., Pfurtscheller, G.: An application framework for controlling an avatar in a desktop based virtual environment via a software SSVEP braincomputer interface. Presence: Teleoperators and Virtual Environments. 19(1), 25-34 (2010)

21. Fellner, D., Havemann, S., Hopp, A.: Dave - eine neue technologie zur preiswerten und hochqualitativen immersiven 3d-darstellung. In: R. Möller (ed.) Proc. 8. Workshop: Sichtsysteme - Visualisierung in der Simulationstechnik, pp. 77-83. Shaker Verlag, Bremen (2003)

22. Friedman, D., Donenfeld, A., Zafran, E.: Neurophysiology-based art in immersive virtual reality. Int'1 J. of Arts and Technology 2(4), 331-347 (2009)

23. George, L., Lécuyer, A.: An overview of research on passive brain-computer interfaces for implicit human-computer interaction. In: International Conference on Applied Bionics and Biomechanics (2010)

24. Groenegress, C., Holzner, C., C.Guger, Slater, M.: Effects of P300-based BCI use on reported presence in a virtual environment. Presence - Teleoperators and Virtual Environments 19(1), $1-11(2010)$

25. Grychtol, B., Lakany, H., Valsan, G., Conway, B.A.: Human behavior integration improves classification rates in real-time bci. IEEE Trans Neural Syst Rehabil Eng 18(4), 362-368 (2010)

26. Guger, C., Daban, S., Sellers, E., Holzner, C., Krausz, G., Carabalona, R., Gramatica, F., Edlinger, G.: How many people are able to control a P300-based brain-computer interface (BCI)? Neuroscience Letters 462(1), 94-98 (2009)

27. Guger, C., Edlinger, G., Krausz, G.: Recent Advances in Brain-Computer Interface Systems, chap. Hardware/Software Components and Applications of BCIs. InTech (2011)

28. Guger, C., Holzner, C., Groenegress, C., Edlinger, G., Slater, M.: Control of a smart home with a brain-computer interface. In: 4th International Brain-Computer Interface Workshop, pp. 339-342 (2008)

29. Guger, C., Holzner, C., Groenegress, C., Edlinger, G., Slater, M.: Brain-computer interface for virtual reality control. In: Proceedings of ESANN 2009, pp. 443-448 (2009) 
30. Guger, C., Schlögl, A., Neuper, C., Walterspacher, D., Strein, T., Pfurtscheller, G.: Rapid prototyping of an EEG-based brain-computer interface (BCI). IEEE Trans. Rehab. Eng 9(1), 49-58 (2001)

31. Haggard, P.: Conscious intention and motor cognition. Trends Cogn Sci 9(6), 290-5 (2005)

32. Kronegg, J., Chanel, G., Voloshynovskiy, S., Pun, T.: EEG-based synchronized braincomputer interfaces: A model for optimizing the number of mental tasks. IEEE Transactions on Neural Systems and Rehabilitation Engineering 15(1), 50-58 (2007)

33. Krusienski, D., Sellers, E., Cabestaing, F., Bayoudh, S., McFarland, D., Vaughan, T., Wolpaw, J.: A comparison of classification techniques for the P300 speller. Journal of Neural Engineering 3, 299-305 (2006)

34. Kuebler, A., Nijboer, F., Mellinger, J., Vaughan, T.M., Pawelzik, H., Schalk, G., McFarland, D.J., Birbaumer, N., Wolpaw, J.R.: Patients with ALS can use sensorimotor rhythms to operate a brain-computer interface. Neurology 64(10), 1775-7 (2005)

35. Lalor, E., Kelly, S., Finucane, C., Burke, R., Smith, R., Reilly, R.B., McDarby, G.: Steadystate vep-based brain computer interface control in an immersive 3-d gaming environment. EURASIP Journal on Applied Signal Processing 19, 3156-3164 (2005)

36. Lécuyer, A.: Using eyes, hands, and brain for 3D interaction with virtual environments: A perception-based approach. Tech. rep., Habilitation thesis (2010)

37. Lécuyer, A., Lotte, F., Reilly, R., Leeb, R., Hirose, M., Slater, M.: Brain-computer interfaces, virtual reality and videogames. IEEE Computer 41(10), 66-72 (2008)

38. Leeb, R.: Brain-computer communication: the motivation, aim, and impact of virtual feedback. Ph.D. thesis, Graz University of Technology (2008)

39. Leeb, R., Friedman, D., Müller-Putz, G.R., Scherer, R., Slater, M., Pfurtscheller, G.: Selfpaced (asynchronous) BCI control of a wheelchair in virtual environments: a case study with a tetraplegics. Computational Intelligence and Neuroscience 2007, 79,642 (2007)

40. Leeb, R., Keinrath, C., Friedman, D., Guger, C., Scherer, R., Neuper, C., Garau, M., Antley, A., Steed, A., Slater, M., Pfurtscheller, G.: Walking by thinking: the brainwaves are crucial, not the muscles! Presence: Teleoperators and Virtual Environments 15, 500-514 (2006)

41. Leeb, R., Lee, F., Keinrath, C., Scherer, R., Bischof, H., Pfurtscheller, G.: Brain-computer communication: motivation, aim and impact of exploring a virtual apartment. IEEE Transactions on Neural Systems and Rehabilitation Engineering 15, 473-482 (2007)

42. Leeb, R., Scherer, R., Friedman, D., Lee, F.Y., Keinrath, C., Bischof, H., Slater, M., Pfurtscheller, G.: Combining BCI and virtual reality: scouting virtual worlds. In: G. Dornhege, J. Millán, T. Hinterberger, D.J. McFarland, K.R. Müller (eds.) Toward brain-computer interfacing, chap. 23, pp. 393-408. MIT Press (2007)

43. Leeb, R., Settgast, V., Fellner, D.W., Pfurtscheller, G.: Self-paced exploring of the Austrian National Library through thoughts. International Journal of Bioelectromagnetism 9, 237-244 (2007)

44. Legény, J., Viciana-Abad, R., Lécuyer, A.: Navigating in virtual worlds using a self-paced SSVEP-based brain-computer interface with integrated stimulation and real-time feedback. Presence - Teleoperators and Virtual Environments (in press)

45. Lotte, F.: Brain-computer interfaces for 3D games: Hype or hope? In: Foundations of Digital Games, pp. 325-327 (2011)

46. Lotte, F., Langhenhove, A.V., Lamarche, F., Ernest, T., Renard, Y., Arnaldi, B., Lécuyer, A.: Exploring large virtual environments by thoughts using a brain-computer interface based on motor imagery and high-level commands. Presence: teleoperators and virtual environments 19(1), 54-70 (2010)

47. Lotte, F., Renard, Y., Lécuyer, A.: Self-paced brain-computer interaction with virtual worlds: a qualitative and quantitative study 'out-of-the-lab'. In: 4th International Brain-Computer Interface Workshop and Training Course, pp. 373-378 (2008)

48. Mason, S., Kronegg, J., Huggins, J., Fatourechi, M., Schloegl, A.: Evaluating the performance of self-paced BCI technology. Tech. rep., Neil Squire Society (2006)

49. Millán, J.d.R., Rupp, R., Müller-Putz, G., Murray-Smith, R., Giugliemma, C., Tangermann, M., Kübler, A., Leeb, R., Neuper, C., Müller, K.R., Mattia, D.: Combining Brain-Computer 
Interfaces and Assistive Technologies: State-of-the-Art and Challenges. Frontiers in Neuroprosthetics (2010)

50. Neuper, C., Scherer, R., Wriessnegger, S., Pfurtscheller, G.: Motor imagery and action observation: modulation of sensorimotor brain rhythms during mental control of a brain-computer interface. Clin Neurophysiol 120(2), 239-247 (2009)

51. Nijholt, A., Bos, D.P.O., Reuderink, B.: Turning shortcomings into challenges: Braincomputer interfaces for games. Entertainment Computing 1(2), 85-94 (2009)

52. Nijholt, A., Tan, D., Pfurtscheller, G., Brunner, C., del R. Millán, J., Allison, B., Graimann, B., Popescu, F., Blankertz, B., Müller, K.R.: Brain-computer interfacing for intelligent systems. IEEE Intelligent Systems 23, 72-79 (2008)

53. Oishi, K., Kasai, T., Maeshima, T.: Autonomic response specificity during motor imagery. J Physiol Anthropol Appl Human Sci 19(6), 255-61 (2000)

54. Pfurtscheller, G., Allison, B., Bauernfeind, G., Brunner, C., Solis Escalante, T., Scherer, R., Zander, T., Müller-Putz, G., Neuper, C., Birbaumer, N.: The hybrid BCI. Front. Neurosci. 4, $42(2010)$

55. Pfurtscheller, G., Leeb, R., Friedman, D., Slater, M.: Centrally controlled heart rate changes during mental practice in immersive virtual environment: a case study with a tetraplegic. International Journal of Psychophysiology 68, 1-5 (2008)

56. Pfurtscheller, G., Leeb, R., Keinrath, C., Friedman, D., Neuper, C., Guger, C., Slater, M.: Walking from thought. Brain Res 1071(1), 145-152 (2006)

57. Pfurtscheller, G., Leeb, R., Slater, M.: Cardiac responses induced during thought-based control of a virtual environment. International Journal of Psychophysiology 62, 134-140 (2006)

58. Pfurtscheller, G., Lopes da Silva, F.H.: Event-related EEG/MEG synchronization and desynchronization: basic principles. Clinical Neurophysiology 110, 1842-1857 (1999)

59. Pfurtscheller, G., Müller-Putz, G.R., Schlögl, A., Graimann, B., Scherer, R., Leeb, R., Brunner, C., Keinrath, C., Lee, F., Townsend, G., Vidaurre, C., Neuper, C.: 15 years of BCI research at Graz University of Technology: current projects. IEEE Transactions on Neural Systems and Rehabilitation Engineering 14, 205-210 (2006)

60. Pfurtscheller, G., Neuper, C.: Motor imagery and direct brain-computer communication. Proc. IEEE 89, 1123-1134 (2001)

61. Pineda, J.A., Silverman, D.S., Vankov, A., Hestenes, J.: Learning to control brain rhythms: making a brain-computer interface possible. IEEE Transactions on Neural Systems and Rehabilitation Engineering 11, 181-184 (2003)

62. Plass-Oude Bos, D., Duvinage, M., Oktay, O., Delgado Saa, J., Guruler, H., Istanbullu, A., Van Vliet, M., Van de Laar, B., Poel, M., Roijendijk, L., Tonin, L., Bahramisharif, A., Reuderink, B.: Looking around with your brain in a virtual world. In: IEEE Symposium on Computational Intelligence, Cognitive Algorithms, Mind, and Brain (SSCI'2011 CCMB) (2011)

63. Renard, Y., Lotte, F., Gibert, G., Congedo, M., Maby, E., Delannoy, V., Bertrand, O., Lécuyer, A.: OpenViBE: An open-source software platform to design, test and use brain-computer interfaces in real and virtual environments. Presence: teleoperators and virtual environments 19(1), 35-53 (2010)

64. Ron-Angevin, R., Diaz-Estrella, A.: Brain-computer interface: Changes in performance using virtual reality technique. Neuroscience Letters 449(2), 123-127 (2009)

65. Ron-Angevin, R., Diaz-Estrella, A., Velasco-Alvarez, F.: A two-class brain computer interface to freely navigate through virtual worlds. Biomedizinische Technik/Biomedical Engineering 54(3), 126-133 (2009)

66. Scherer, R., Lee, F., Schlögl, A., Leeb, R., Bischof, H., Pfurtscheller, G.: Towards self-paced brain-computer communication: Navigation through virtual worlds. IEEE, Transactions on Biomedical Engineering 55(2), 675-682 (2008)

67. Scherer, R., Schlögl, A., Lee, F., Bischof, H., Jansa, J., Pfurtscheller, G.: The self-paced graz brain-computer interface: methods and applications. Computational Intelligence and Neuroscience 2007(Article ID 79826), 1-9 (2007)

68. Slater, M., Usoh, M., Steed, A.: Taking steps: the influence of a walking technique on presence in virtual reality. ACM Trans. Comput.-Hum. Interact. 2(3), 201-219 (1995) 
69. Taylor, R.M., Hudson, T.C., Seeger, A., Weber, H., Juliano, J., Helser, A.: VRPN: a deviceindependent, network-transparent VR peripheral system. In: VRST '01: Proceedings of the ACM symposium on Virtual reality software and technology, pp. 55-61. ACM Press, New York, NY, USA (2001)

70. Touyama, H.: Advances in Human Computer Interaction, chap. Brain-CAVE Interface Based on Steady-State Visual Evoked Potential, pp. 437-450. No. 26 in ISBN 978-953-7619-15-2. InTech Education and Publishing (2008)

71. Vaughan, T.M., Wolpaw, J.R., Donchin, E.: EEG-based communication: prospects and problems. IEEE transactions on rehabilitation engineering 4, 425-430 (1996)

72. Velasco-Álvarez, F., Ron-Angevin, R.: Free virtual navigation using motor imagery through an asynchronous brain-computer interface. Presence: teleoperators and virtual environments 19(1), 71-81 (2010)

73. Vialatte, F., Maurice, M., Dauwels, J., Cichocki, A.: Steady-state visually evoked potentials: Focus on essential paradigms and future perspectives. Progress in Neurobiology, 90, 418-438 (2010)

74. Williamson, J., Murray-Smith, R., Blankertz, B., Krauledat, M., Müller, K.: Designing for uncertain, asymmetric control: Interaction design for brain-computer interfaces. International Journal of Human Computer Studies 67(10), 827-841 (2009)

75. Wolpaw, J., Birbaumer, N., McFarland, D., Pfurtscheller, G., Vaughan, T.: Brain-computer interfaces for communication and control. Clinical Neurophysiology 113(6), 767-791 (2002)

76. Zander, T., Kothe, C.: Towards passive brain-computer interfaces: applying brain-computer interface technology to human-machine systems in general. Journal of Neural Engineering 8 (2011)

77. Zickler, C., Donna, V.D., Kaiser, V., Al-Khodairy, A., Kleih, S., Kuebler, A., Malavasi, M., Mattia, D., Mongardi, S., Neuper, C., Rohm, M., Rupp, R.: Brain computer interaction applications for people with disabilities: Defining user needs and user requirements. In: AAATE (2009) 